\title{
Silencing of suppressor of cytokine signaling 1 enhances the immunological effect of mucin 1-calreticulin-primed 4T1 cell-treated dendritic cells in breast cancer treatment
}

\author{
SONG QIN ${ }^{1}$, ZHIPENG GAO ${ }^{1}$, YU LIU $^{2,3}, \mathrm{CHANGBAI} \mathrm{LIU}^{2}, \mathrm{JUN}_{\mathrm{WANG}}{ }^{1 *}$ and LI LI ZOU ${ }^{1,2^{*}}$ \\ ${ }^{1}$ Translational Neuroscience and Neural Regeneration and Repair Institute/Institute of Cell Therapy, \\ The First Hospital of Yichang, China Three Gorges University, Yichang, Hubei 443002; \\ ${ }^{2}$ Department of Microbiology and Immunology, Medical College, China Three Gorges University, Yichang, \\ Hubei 443000; ${ }^{3}$ Department of Oncology, Gong'an County Hospital, Yichang, Hubei 434300, P.R. China
}

Received October 18, 2016; Accepted September 28, 2017

DOI: $10.3892 / \mathrm{ol} .2017 .7477$

\begin{abstract}
In cancer immunotherapy, dendritic cell (DC)-based vaccines represent a promising, yet challenging, treatment method. In addition to overcoming the low expression levels of antigenic epitopes on cancer cells, it is also necessary to overcome the inhibitory effect of suppressor of cytokine signaling 1 (SOCS1) on DC self-antigen presentation. Our group previously demonstrated that calreticulin (CRT) translocated type I transmembrane glycoprotein mucin 1 (MUC1), a breast cancer antigen, to the surface of $4 \mathrm{~T} 1$ cells, and that treatment with MUC1-CRT-primed 4T1 cell-treated DCs induced apoptosis in a breast cancer cell line. In the present study, cell penetrate peptide, hpp10-DRBD was successfully used to deliver siRNAs into bone marrow-derived (BM) DCs to construct SOCS1-silenced DCs, which were incubated with MUC1-CRT-primed 4T1 cells, and antigen-specific antitumor immunity was markedly enhanced in vitro and in vivo. These results demonstrated that SOCS1-silencing, combined with MUC1-CRT-primed 4T1 cell treatment, may induce increased cytokine production and T cell proliferation by DCs. Furthermore, the in vivo experimental data demonstrated that the silencing of SOCS1 combined with MUC1-CRT-primed 4T1 treatment of BMDCs may induce enhanced immunological effects. The results of the present study have implications for the development of more effective DC-based tumor vaccines,
\end{abstract}

Correspondence to: Dr Jun Wang or Dr Li Li Zou, Translational Neuroscience and Neural Regeneration and Repair Institute/Institute of Cell Therapy, The First Hospital of Yichang, China Three Gorges University, 8 Daxue Road, Yichang, Hubei 443002, P.R. China

E-mail: wangj33@mail2.sysu.edu.cn

E-mail: zoulili@ctgu.edu.cn

${ }^{*}$ Contributed equally

Key words: dendritic cells, suppressor of cytokine signaling 1, calreticulin, type I transmembrane glycoprotein mucin 1, vaccine, cancer suggesting that the combination of high tumor-associated antigen expression levels on cancer cells with the silencing of a critical inhibitor of DC antigen presentation may be beneficial.

\section{Introduction}

Dendritic cells (DCs) are antigen-presenting cells (APCs) that serve a critical function in maintaining tolerance to self-antigens and in the induction and regulation of immune responses due to their intrinsic immune-priming abilities. These properties make the development of DC-based vaccines for cancer immunotherapy an attractive prospect $(1,2)$. However, to date, the clinical outcomes of studies investigating DC-based vaccination for the treatment of cancer have not been promising. One of the limitations of this approach is the low expression level of antigenic epitopes on cancer cells, making them poorly immunogenic to DCs (3-6). In cancer immunotherapy, it is essential to sufficiently expose tumor-associated antigens (TAAs) to DCs in order to stimulate DC maturation and facilitate the subsequent activation of antigen-specific immune responses. Our previous data revealed that calreticulin (CRT) is a key molecule involved in cell recognition and that it may act as an 'immunological adjuvant' by translocating type I transmembrane glycoprotein mucin 1 (MUC1), a breast cancer antigen, to the surface of 4T1 cells (7). The MUC1-CRT-primed 4T1 cells were recognized by DCs, leading to a specific antitumor immune response.

Therefore, efforts to develop tumor vaccines have focused on promoting the maturation of DCs as a means of enhancing antitumor immunity (8-10). However, the induction of immunity against cancer cells is also restricted by intrinsic inhibitory mechanisms; for example, suppressor of cytokine signaling 1 (SOCS1) functions as a negative regulator of Janus kinases (JAKs), thereby acting as an inhibitory regulator of antigen presentation by DCs and the magnitude of adaptive immune responses (11). The present study specifically focused on the incubation of SOCS1-silenced DCs with MUC1-CRT-primed 4T1 cells in an attempt to enhance specific immunological effects in vitro and in vivo. RNA interference technology, which is widely used to silence endogenous molecules involved 
in DC-mediated apoptosis and immunosuppressive signaling, was utilized to improve responses against cancer cells $(12,13)$. In the present study, small interfering RNAs (siRNAs) were effectively introduced into DCs with a simple and controllable antigen delivery system: A novel, human-derived, positively-charged cell-penetrating peptide, denoted hpp10 (14), accompanied and shielded the negatively-charged siRNA via a double-stranded RNA-binding domain (DRBD) (15).

The present study has implications for the development of more effective DC-based breast cancer vaccines by combining high TAA expression levels on breast cancer cells with silencing of the critical inhibitor of antigen presentation on DCs. Therefore, the results of the present study may lead to an improved antitumor immunotherapeutic approach for the treatment of breast cancer.

\section{Materials and methods}

Experimental animals. Approval from the Medical Animal Care and Welfare Committee of China Three Gorges University (Hubei, China) was obtained prior to any experimentation using animals. A total of 97 female BALB/c mice (18 $\pm 2 \mathrm{~g}, 4-6$ weeks old) were purchased from the Laboratory Animal Center of China Three Gorges University. All mice were housed in specific pathogen-free conditions, with free access to food and water. The ambient temperature was maintained at $22 \pm 2^{\circ} \mathrm{C}$ with a humidity of $50-60 \%$ and a $12 \mathrm{~h}$ light/dark cycle. All experiments in mice were performed following the relevant institutional guidelines and regulations, and were subject to a protocol approved by the China Three Gorges University Animal Care Committee.

Drugs and chemicals. Lipofectamine 2000 was purchased from Thermo Fisher Scientific, Inc. (Waltham, MA, USA). Lipopolysaccharide (LPS) and anti-signal transducer and activator of transcription 1 (STAT1) antibodies were purchased from Sigma-Aldrich; Merck KGaA (Darmstadt, Germany; cat. nos. L2630 and SAB4300133, respectively); the Lactate Dehydrogenase (LDH) Activity Assay kit was also purchased from Sigma-Aldrich; Merck KGaA. Mitoxantrone (MIT) was purchased from Jiangsu Aosaikang Pharmaceutical Co., Ltd. (Nanjing, China). Magnetic beads and anti-SOCS1 antibodies (cat. no. 38-5200) were purchased from eBioscience; Thermo Fisher Scientific, Inc. (Waltham, MA, USA). Mouse granulocyte-macrophage colony-stimulating factor (mGM-CSF) and mouse interleukin-4 (mIL-4) primary antibodies were purchased from PeproTech, Inc. (Rocky Hill, NJ, USA; cat. nos. 315-03 and 500-p45, respectively), which were used for DC cell culture as described previously (7). Anti-cluster of differentiation (CD)11c-allophycocyanin, anti-CD83-fluorescein isothiocyanate and anti-phosphorylated (p)-STAT1 antibodies were purchased from Biolegend, Inc. (San Diego, CA, USA; cat. No. 117305, cat. nos. 121505 and 686402, respectively). Purified anti- $\beta$-actin antibody was purchased from Biolegend, Inc. (San Diego, CA, USA; cat. no. 643801). Horseradish peroxidase (HRP)-labeled goat anti-mouse immunoglobulin $\mathrm{G}$ (IgG) [heavy and light $(\mathrm{H}+\mathrm{L})]$ and HRP-labeled goat anti-rabbit IgG $(\mathrm{H}+\mathrm{L})$ were purchased from Beyotime Co. (Shanghai, China; cat. nos. A0216 and A0208, respectively). Interferon (IFN) $-\gamma$ and tumor necrosis factor (TNF)- $\alpha$ ELISA kits were purchased from Boster Biological Technology (Pleasanton, CA, USA; cat. nos. EK0375 and EK0527, respectively). Lymphocyte separation medium was purchased from Dakewe. Co. (Shenzhen, China; cat. no. DKW33-R0100). RPMI-1640 medium, L-glutamine, streptomycin and penicillin were all purchased from Biosera, Inc. (Nuaille, France). Bovine serum albumin and fetal bovine serum (FBS) and beta-actin were purchased from Gibco; Thermo Fisher Scientific, Inc. All primers were synthesized by Sangon Biotech Co., Ltd. (Shanghai, China).

Cell line culture. Bone marrow-derived dendritic cells (BMDCs) were collected and identified as previously described (7). The DC2.4 cell line was purchased from Suershengwu Co., Ltd. (Shanghai, China), the breast cancer 4T1 cell line, originating from BALB/c mice, was purchased from American Type Culture Collection (Manassas, VA, USA) and all cells were maintained in the laboratory in the China Three Gorges University. Cells were cultured $\left(2.0 \times 10^{5} / \mathrm{ml}\right)$ in complete medium (RPMI-1640 medium supplemented with $10 \mathrm{mmol} / \mathrm{l}$ L-glutamine, $10 \%$ heat-inactivated FBS, $100 \mathrm{U} / \mathrm{ml}$ penicillin and $100 \mu \mathrm{g} / \mathrm{ml}$ streptomycin) in a humidified incubator at $37^{\circ} \mathrm{C}$ containing $5 \% \mathrm{CO}_{2}$.

SOCS1 siRNA selection. Three siRNAs targeting SOCS1 (and a negative control) were synthesized by Shanghai GenePharma Co., Ltd. (Shanghai, China), the sequences of which are listed in Table I. The RNAs were de-protected and annealed according to the manufacturer's protocol. DC2.4 cells were seeded onto 6-well culture plates $\left(1.0 \times 10^{6} /\right.$ well) and were cultivated to semi-confluence in RPMI-1640 medium for $24 \mathrm{~h}$ at $37^{\circ} \mathrm{C}$. Cells were further incubated with $1.0 \mu \mathrm{g}$ SOCS1 siRNA and $3.0 \mu \mathrm{l}$ Lipofectamine 2000 reagent in $100 \mu \mathrm{l}$ serum-free medium for $4-6 \mathrm{~h}$ at $37^{\circ} \mathrm{C}$. Subsequently, the cells were lysed in whole-cell extraction buffer $(50 \mathrm{mM}$ Tris/ $\mathrm{HCl}, \mathrm{pH} \mathrm{7.4,}$ $250 \mathrm{mM} \mathrm{NaCl}, 5 \mathrm{mM}$ EDTA, $50 \mathrm{mM} \mathrm{NaF}, 1 \mathrm{mM} \mathrm{Na} \mathrm{VO}_{4}, 1 \%$ Nonidet P40, $0.02 \% \mathrm{NaN}_{3}$ and $1 \mathrm{mM}$ phenylmethylsulfonyl fluoride). Following centrifugation at $10,000 \mathrm{x}$ g for $20 \mathrm{~min}$ at $4^{\circ} \mathrm{C}$, the supernatant was collected and the protein concentration was quantified by a BCA assay. Total protein (40 $\mu \mathrm{g}$ per lane) was used for western blot analyses and was probed with primary antibodies specific to SOCS1, pSTAT1 and STAT1 according to a protocol described previously (16).

To measure cytokine production, splenic lymphocytes were separated and collected in a lymphocyte separation medium, as described previously (17), and successful purification was verified by light field microscopy (magnification, x40). DC2.4 cells were incubated with $1.0 \mu \mathrm{g}$ SOCS1 siRNAs in $100 \mu \mathrm{l}$ serum-free medium for $1 \mathrm{~h}$ at $37^{\circ} \mathrm{C}$. To stimulate DCs, $2 \times 10^{5}$ cells $/ \mathrm{ml}$ were cultured in the presence of $100 \mathrm{ng} / \mathrm{ml}$ LPS for $24 \mathrm{~h}$ and were further incubated with lymphocytes at $37^{\circ} \mathrm{C}$ in $5 \% \mathrm{CO}_{2}$ for $72 \mathrm{~h}$. Following stimulation, IFN- $\gamma$ levels in the culture supernatants (100 $\mu \mathrm{l} /$ well) were analyzed using an ELISA kit in accordance with the manufacturer's protocol.

Cellular uptake of hpp10-DRBD-SOCS1 siRNA. To efficiently deliver SOCS1 siRNA into BMDCs, hpp10-DRBD was expressed and purified. Briefly, hpp10-linker-DRBD was synthesized by Shanghai Shenggong Biology Engineering Technology Service, Ltd. (Shanghai, China), and was linked 
Table I. SOCS1 siRNAs and hpp10-DRBD sequences.

Name

Sequences (5'-3')

Scramble control

SOCS1 siRNA1

SOCS1 SiRNA2

SOCS1 siRNA3

Hpp10-DRBD

\begin{abstract}
FAM-UUCUCCGAAGGUGUCACGU
FAM-AGACCUUCGACUGCCUUUUTT

FAM-CUACCUGAGUUCCUUCCCCTT

FAM-ACACUCACUUCCGCACCUUTT

AAAATCCCCCTGCCCCGCTTCAAACTGAAATGTATCTTCTGTAAGAAGCGGAGGAAA AGACTCGAGGGCGATCCGGCTGGTGATCTTTCAGCAGGTTTCTTCATGGAGGAACTT AATACATACCGTCAGAAGCAGGGAGTAGTACTTAAATATCAAGAACTGCCTAATTCAG GACCTCCACATGATAGGAGGTTTACATTTCAAGTTATAATAGATGGAAGAGAATTTCC AGAAGGTGAAGGTAGATCAAAGAAGGAAGCAAAAATGCCGCAGCCAAATTAGCTGT TGAGATACTTAATAAGGAAAAGAAGGCAGGATCC
\end{abstract}

Underlined sections represent $X$ hoI and BamHI, respectively. The section in bold represents the linker sequence, preceded by the Hpp 10 sequence and followed by the DRBD sequence. SOCS1, suppressor of cytokine signaling 1; siRNA, small interfering RNA; DRBD, double-stranded RNA binding-domain.
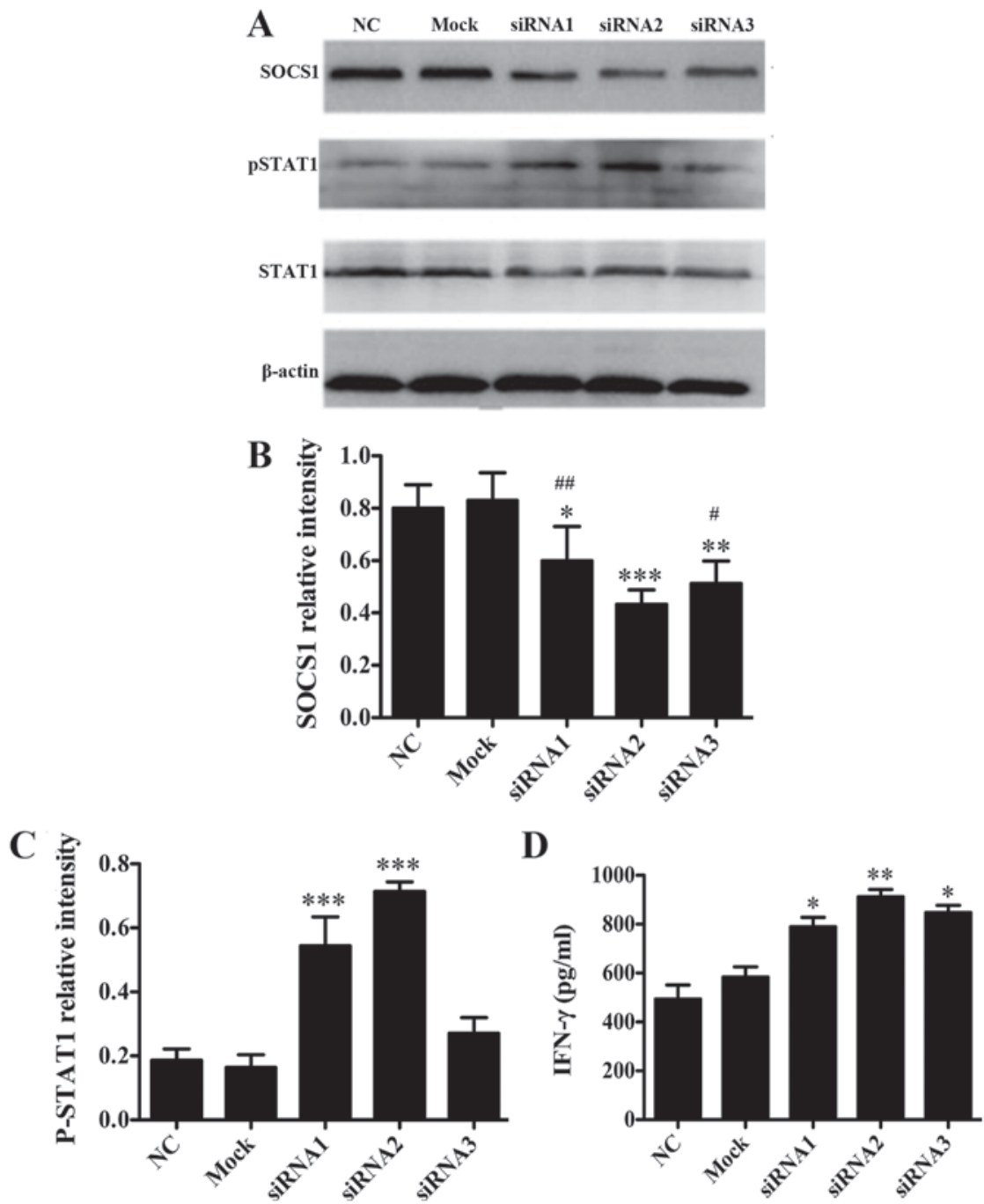

Figure 1. SOCS1 negatively regulates DCs in vitro. (A) DC2.4 cells were transfected with SOCS1 siRNAs (siRNA1-3) using Lipofectamine 2000. The protein expression levels of SOCS1, pSTAT1 and STAT1 in DC2.4 cells $48 \mathrm{~h}$ after transfection with SOCS1 siRNA were determined by western blot analysis. Representative western blot analysis results from one of three independent experiments are demonstrated. The intensity of (B) SOCS1 and (C) pSTAT1 bands were normalized to that of the $\beta$-actin bands, and the relative intensities (ratios) are presented. (D) Levels of IFN- $\gamma$ secretion by siRNA-transfected or mock-transfected DC2.4 cells in response to $100 \mathrm{ng} / \mathrm{ml}$ LPS-simulation for $24 \mathrm{~h} .{ }^{*} \mathrm{P}<0.05,{ }^{* *} \mathrm{P}<0.01$ and ${ }^{* * * *} \mathrm{P}<0.001$ vs. NC; ${ }^{*} \mathrm{P}<0.05$ and ${ }^{\# \#} \mathrm{P}<0.01$ vs. siRNA2 group. SOCS1, suppressor of cytokine signaling 1; DCs, dendritic cells; siRNA, small interfering RNA; pSTAT1, phosphorylated signal transducer and activator of transcription 1 ; IFN- $\gamma$, interferon- $\gamma$; LPS, lipopolysaccharide. 
Table II. Primer pairs.

\begin{tabular}{lll}
\hline Primer name & \multicolumn{1}{c}{ Sequence (5'-3') } & Restriction enzyme \\
\hline CRT_F & GGTTCTGTCGACGACCCTGCCATCTATTTC & SalI \\
CRT_R & TACGGATCCCTACAGCTCATCCTTGGC & BamHI \\
MUC1_F & AATAGTCGACCCGGACACCAGGCCGGCCCC & SalI \\
MUC1_R & ATATGGATCCGGCCGAGGTGACACCATGGG & BamHI \\
MUC1-CRT_F & GGTCGTCGACAGAACCGCCGGCCGAGG & SalI \\
MUC1-CRT_R & GGTTCTGGATCCGACCCTGCCATCTATTTC & BamHI \\
\hline
\end{tabular}

Underlined sequences represent the restriction enzyme site mentioned in the third column. CRT, calreticulin; F, forward; R, reverse; MUC1, type I transmembrane glycoprotein mucin 1.

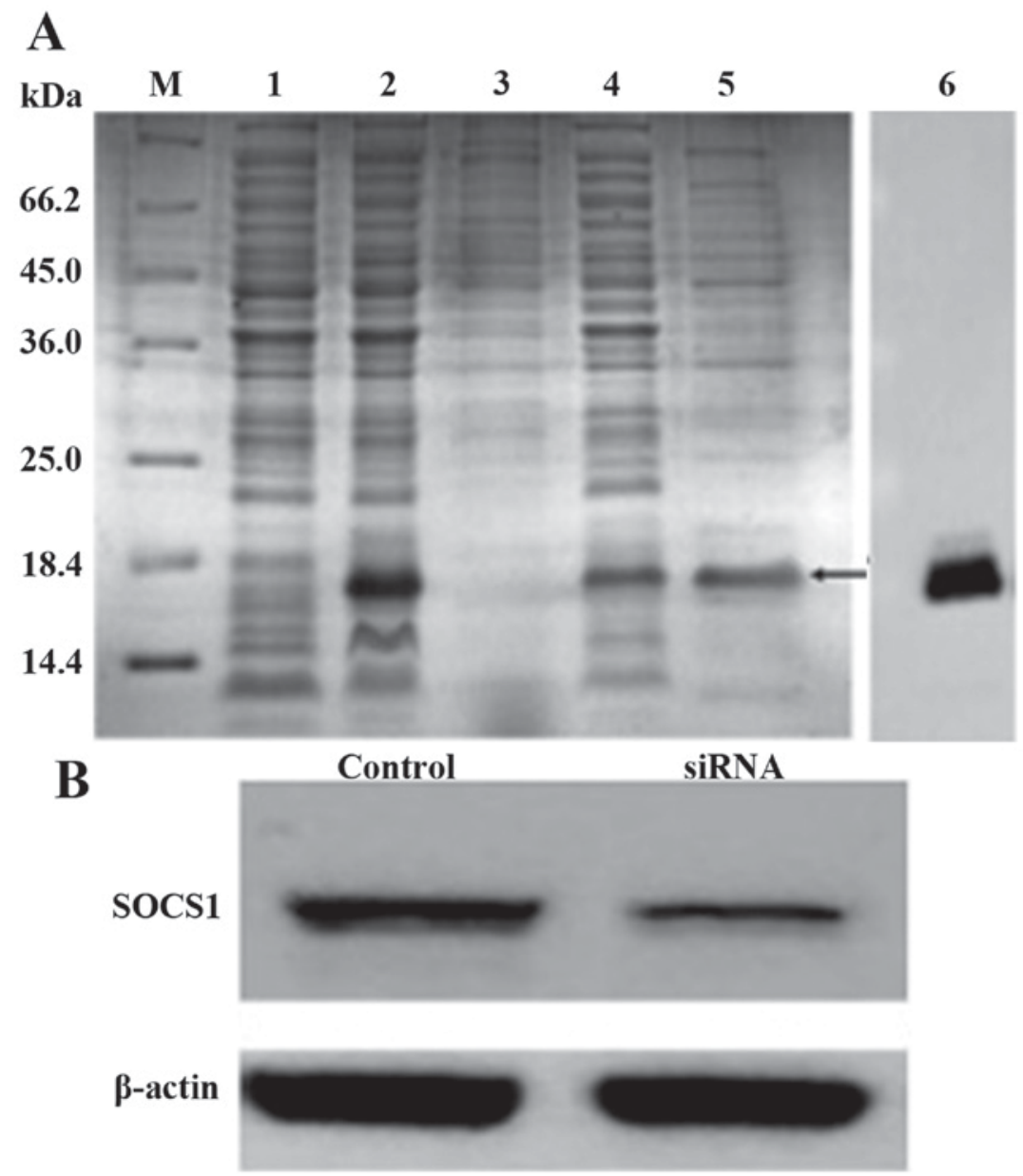

Figure 2. Hpp10-DRBD construction and SOCS1 siRNA delivery. (A) SDS-PAGE and Coomassie Blue staining of purified recombinant hpp10-DRBD. M, protein marker 431; 1, non-induced protein; 2, induced protein; 3, precipitate; 4, supernatant; 5, eluted protein; and 6, western blotting to detect the purified protein. (B) Western blot analysis was performed to detect SOCS1 expression levels in BMDCs following transfection with hpp10-DRBD-SOCS1 siRNA for 48 h. DRBD, double-stranded RNA binding-domain; SOCS1, suppressor of cytokine signaling 1; siRNA, small interfering RNA; BMDC, bone marrow-derived dendritic cell.

to compatible sites (XhoI/BamHI) of a pET15b vector (Preclinical Medicine Key Laboratory of China Three Gorges University) to yield recombinant protein hpp10-DRBD (Table I). hpp10-DRBD was expressed using isopropyl $\beta$-D-1-thiogalactopyranoside induction and was purified under native conditions in its soluble form, as described previously (18).
The internalization of hpp10-DRBD-SOCS1 siRNA into BMDCs was observed using fluorescence microscopy (magnification, x40). Cells were plated onto6-well-plates $\left(1.0 \times 10^{6} /\right.$ well $)$ and were cultured to semi-confluence in RPMI-1640 medium for $24 \mathrm{~h}$ at $37^{\circ} \mathrm{C}$. Cells were washed three times with $\mathrm{PBS}$, followed by incubation with $10.0 \mu \mathrm{g}$ hpp10-DRBD fusion protein and $1.0 \mu \mathrm{g}$ selected SOCS1 
A

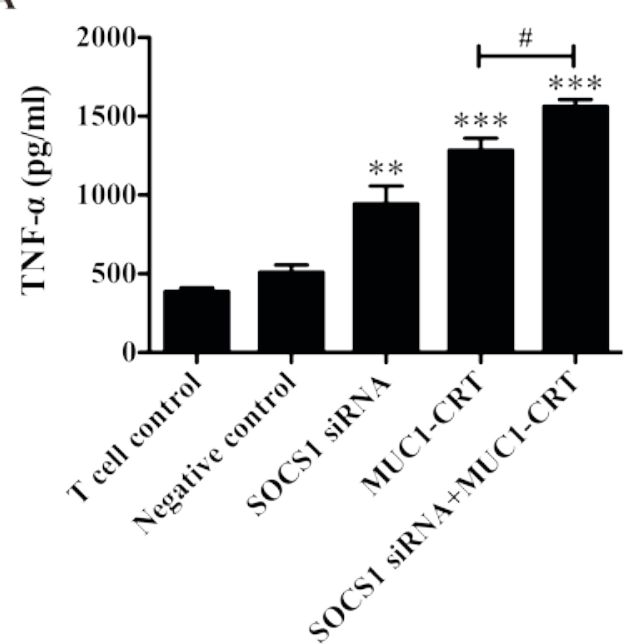

B

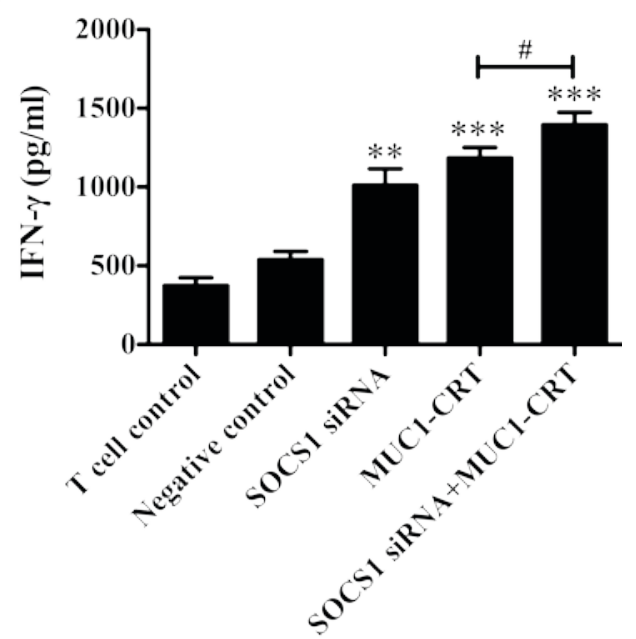

C

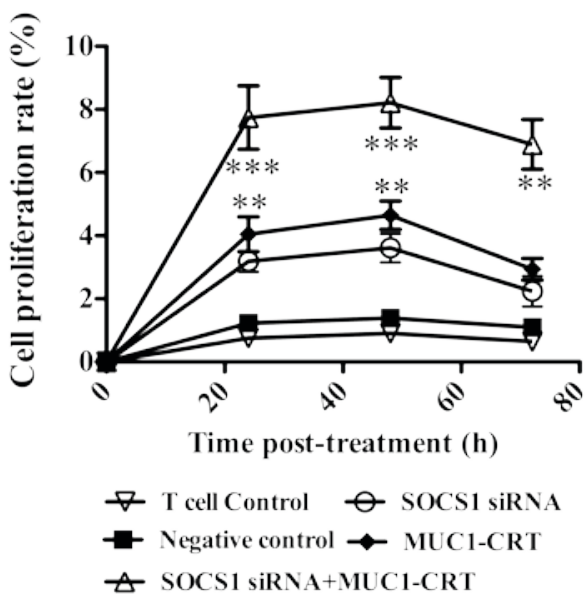

D

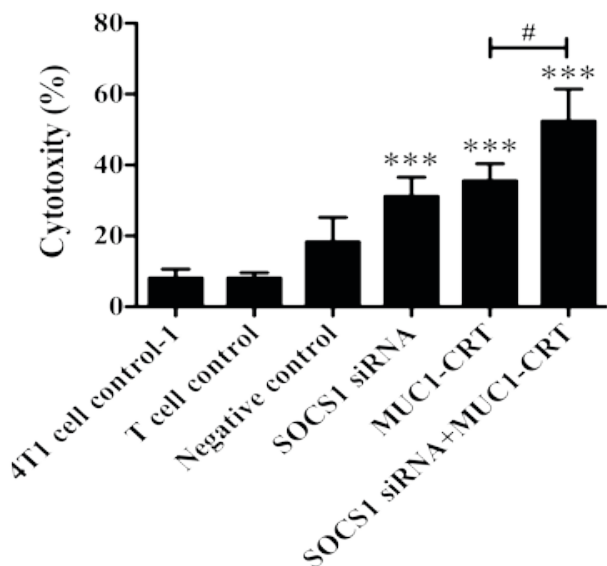

Figure 3. Silencing of SOCS1 enhances the immunological effects of MUC1-CRT-primed 4T1-treated DCs in vitro. Pre-treated BMDCs were incubated with splenocytes, and culture supernatants (100 $\mu 1 /$ well) were used to analyze production of the cytokines, (A) TNF- $\alpha$ and (B) IFN- $\gamma$, by ELISA assay. (C) Pre-treated BMDCs were incubated with splenocytes at $37^{\circ} \mathrm{C}$ for 24,48 or $72 \mathrm{~h}$, and an MTT assay was used to evaluate T cell proliferation. (D) Pre-treated BMDCs were incubated with splenocytes at $37^{\circ} \mathrm{C}$ for $48 \mathrm{~h}$ and splenocytes were separated by magnetic beads, followed by incubation with $4 \mathrm{~T} 1$ cells at $37^{\circ} \mathrm{C}$ for $30 \mathrm{~min}$. The specific killing activity of cytotoxic $\mathrm{T}$ lymphocytes induced by $\mathrm{DC}$ was evaluated using an $\mathrm{LDH}$ assay. ${ }^{*} \mathrm{P}<0.05$, ${ }^{* *} \mathrm{P}<0.01$ and ${ }^{* * * *} \mathrm{P}<0.001$ vs. $\mathrm{NC} ;{ }^{\#} \mathrm{P}<0.05$. Cell-free supernatant was used to detect membrane integrity by lactate dehydrogenase assay. SOCS1, suppressor of cytokine signaling $1 ;$ MUC1, type I transmembrane glycoprotein mucin 1; CRT, calreticulin; DCs, dendritic cells; BMDCs, bone marrow-derived DCs; TNF- $\alpha$, tumor necrosis factor- $\alpha$; IFN- $\gamma$, interferon- $\gamma$; LDH, lactate dehydrogenase.

siRNA in serum-free medium for $1 \mathrm{~h}$ at $37^{\circ} \mathrm{C}$. The medium was removed and the cells were washed three times with PBS, prior to being imaged using a fluorescence microscope (magnification, x40). Western blot analysis was performed to detect SOCS1 expression levels following treatment with hpp10-DRBD-SOCS1 siRNA using an anti-SOCS1 antibody, as aforementioned.

DC preparation. BMDCs were seeded onto 6-well culture plates $\left(1.0 \times 10^{6} /\right.$ well) in complete medium (RPMI-1640 medium supplemented with $10 \mathrm{mmol} / 1 \mathrm{~L}$-glutamine, $10 \%$ heat-inactivated FBS, $100 \mathrm{U} / \mathrm{ml}$ penicillin and $100 \mu \mathrm{g} / \mathrm{ml}$ streptomycin) in a humidified incubator at $37^{\circ} \mathrm{C}$ containing $5 \% \mathrm{CO}_{2}$ and were divided into groups $\mathrm{A}, \mathrm{B}, \mathrm{C}$ and $\mathrm{D}$, according to the following treatments. For group $\mathrm{A}$, BMDCs were incubated with $10.0 \mu \mathrm{g}$ hpp10-DRBD and $1.0 \mu \mathrm{g}$ SOCS1 siRNA in serum-free medium for $1 \mathrm{~h}$ at $37^{\circ} \mathrm{C}$. For group B, pEGFP-type I transmembrane glycoprotein mucin 1 (MUC1)-CRT was constructed as previously described (7) and the primer pairs are presented in Table II. The pEGFP-MUC1-CRT plasmid $(1.0 \mu \mathrm{g})$ was transfected into 4T1 cells to construct MUC1-CRT-primed 4T1 cells by adding 3.0 $\mu \mathrm{l}$ Lipofectamine 2000 for 4-6 h at $37^{\circ} \mathrm{C}$, and the efficiency was observed using fluorescence microscopy (magnification, x40). Subsequently, $1.0 \times 10^{6}$ MUC1-CRT-primed 4T1 cells were treated with $8 \mu \mathrm{g} / \mathrm{ml}$ MIT for $12 \mathrm{~h}$ to induce CRT and MUC1 translocation to the surface of pre-apoptotic $4 \mathrm{~T} 1$ cells, prior to being further incubated $\left(1.0 \times 10^{6} /\right.$ well at $\left.37^{\circ} \mathrm{C}\right)$ with BMDCs. Cells in group $\mathrm{C}$ were double-treated; BMDCs were incubated with hpp10-DRBD-SOCS1 siRNA followed by incubation with MUC1-CRT-primed 4T1 cells (as described for groups A and B). Finally, group D BMDCs were untreated. All cells were washed three times with PBS and collected.

For the LDH leakage assay and animal experiments, BMDCs from each of the 4 groups were collected separately using magnetic beads according to the manufacturer's protocol. The beads provided a strong magnetic attraction for 

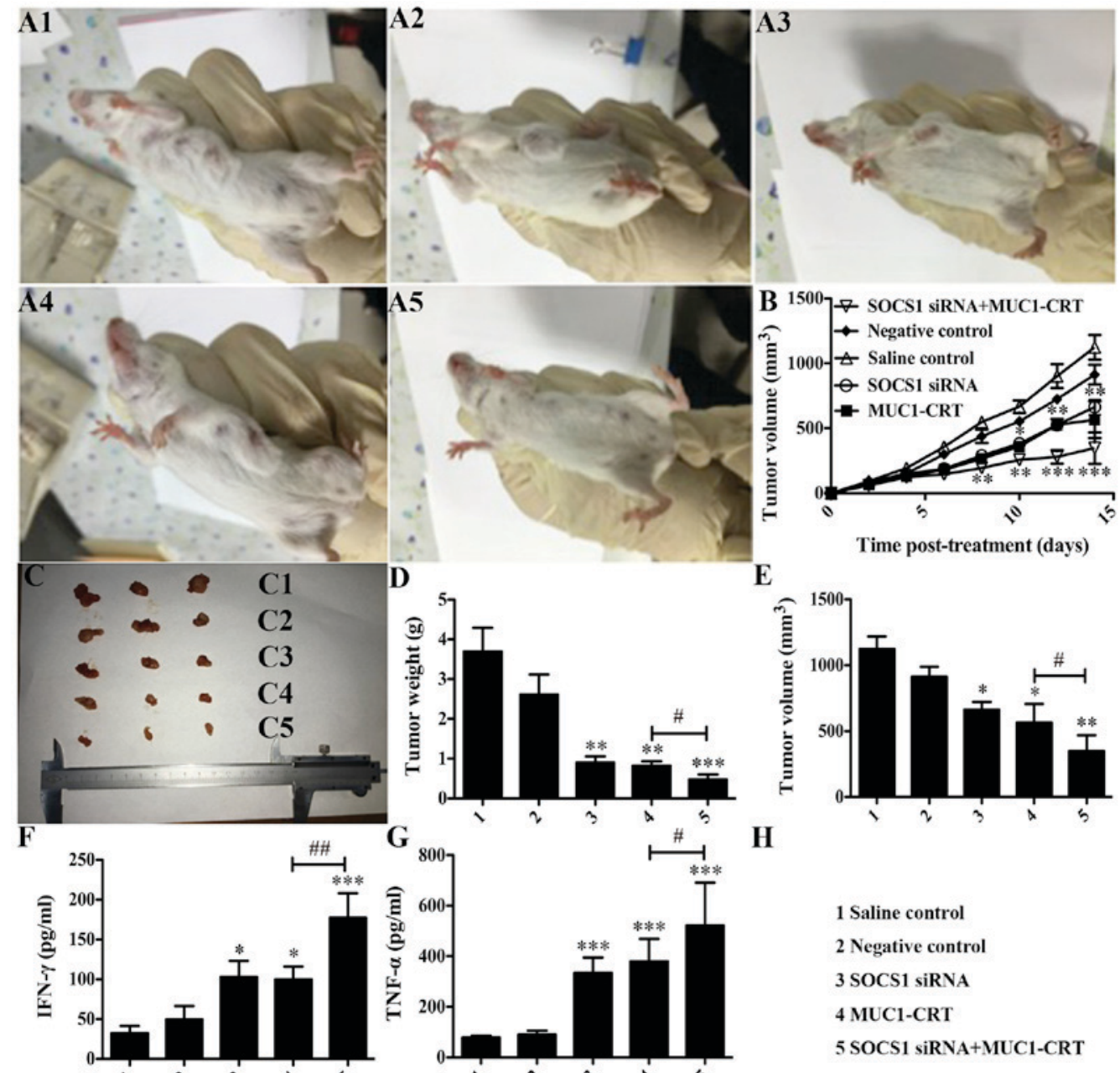

Figure 4. Silencing of SOCS1 enhances the immunological effect of MUC1-CRT-primed 4T1-treated DCs in vivo. Mice were randomly divided into 5 groups ( $\mathrm{n}=10$ per group). Mice in each group were administered with an intradermal injection of $100 \mu \mathrm{l}$ normal saline, $100 \mu 1$ untreated DCs, $100 \mu 1$ SOCS1 siRNA-treated DCs, $100 \mu 1$ MUC1-CRT-primed 4T1-treated DCs or $100 \mu 1$ SOCS1 siRNA- and MUC1-CRT-primed 4T1-treated (double-treated) DCs. (A) Example images demonstrate tumor sizes. (B) Tumor volume $\left(\mathrm{mm}^{3}\right)$ was assessed every other day. Tumor sizes are presented as (C) an image, (D) tumor weight and (E) tumor volume following sacrifice and tumor isolation 16 days after treatment. Secretion of the cytokines, (F) IFN- $\gamma$ and $(\mathrm{G}) \mathrm{TNF}-\alpha$. "P<0.05, ${ }^{* *} \mathrm{P}<0.01$ and ${ }^{* * *} \mathrm{P}<0.001$ vs. NC; ${ }^{\#} \mathrm{P}<0.05,{ }^{\# \#} \mathrm{P}<0.01$. SOCS1, suppressor of cytokine signaling 1 ; siRNA, small interfering RNA; MUC1, type I transmembrane glycoprotein mucin 1 ; CRT, calreticulin; DCs, dendritic cells; IFN- $\gamma$, interferon- $\gamma$; TNF- $\alpha$, tumor necrosis factor- $\alpha$.

efficient separation in standard microcentrifuge tubes, and were adjusted to a concentration of $1.0 \times 10^{7} / \mathrm{ml}$ for further use.

\section{In vitro functional assays}

Cytokine production assays. BMDCs were seeded onto 6-well culture plates $\left(1.0 \times 10^{6} /\right.$ well), and splenocytes were harvested and prepared as single cell suspensions (7). Pre-treated BMDCs (as described under the 'DC preparation' subheading) were incubated with splenocytes at $37^{\circ} \mathrm{C}$ for $72 \mathrm{~h}$. Following centrifugation at $800 \mathrm{x}$ g for $5 \mathrm{~min}$ at room temperature, the supernatant was collected and culture supernatants (100 $\mu \mathrm{l} /$ well) were used to analyze production of the cytokines IFN- $\gamma$ and TNF- $\alpha$ by ELISA, according to the manufacturer's protocol.

T cell proliferation assay. The MTT assay was used to evaluate $\mathrm{T}$ cell proliferation. BMDCs and splenocytes were prepared as aforementioned. Pre-treated BMDCs $\left(5 \times 10^{5}\right.$ cells/well $)$ (as described under the 'DC preparation' subheading) were incubated with splenocytes $\left(5 \times 10^{5}\right.$ cells/well $)$ at $37^{\circ} \mathrm{C}$ for 24 ,
48 or $72 \mathrm{~h}$, and were washed three times with PBS. Cells were incubated in fresh medium containing MTT solution $(5 \mathrm{mg} / \mathrm{ml}$ with PBS) for $4 \mathrm{~h}$ at $37^{\circ} \mathrm{C}$ and absorbance was measured at $540 / 570 \mathrm{~nm}$ using a Multiskan Spectrum plate reader (Thermo Fisher Scientific, Inc.).

LDH leakage assays. Membrane integrity was assessed by $\mathrm{LDH}$ leakage into the culture medium. Pre-treated BMDCs $\left(5 \times 10^{5}\right.$ cells/well) were incubated with splenocytes $\left(5 \times 10^{5}\right.$ cells/well) at $37^{\circ} \mathrm{C}$ for $48 \mathrm{~h}$, and the splenocytes were separated by magnetic beads, according to the manufacturer's protocol. 4T1 cells were harvested and single-cell suspensions were prepared as previously described (7) and subsequently incubated with separated splenocytes at $37^{\circ} \mathrm{C}$ for $16 \mathrm{~h}$. The specific killing activity of cytotoxic $\mathrm{T}$ lymphocytes induced by DC was evaluated using an LDH assay. Cell-free supernatant was obtained and transferred onto 96-well plates, and the LDH substrate reaction buffer, taken from the LDH Activity Assay kit, was added to each well for a 10-min enzymatic reaction according to the manufacturer's protocol Absorbance was 
measured at 560/590 $\mathrm{nm}$ using a Multiskan Spectrum plate reader.

\section{In vivo functional assays}

Immunization with DCs. A total of 50 mice were randomly divided into 5 groups (A, B, C, D and E). To generate a breast cancer-bearing mouse model, the BALB/c mouse breast cancer 4T1 cell line was sub-cultured twice and intradermally injected into the dorsal skin of BALB/c mice $\left(2.0 \times 10^{5}\right.$ cells per injection). After 5 days of growth, a palpable tumor had formed. The mice were then intradermally injected with one of the following: $100 \mu \mathrm{l}$ normal saline (group A), $100 \mu 11.0 \times 10^{7} / \mathrm{ml}$ untreated DCs (group B), $100 \mu 1$ 1.0x107/ml SOCS1 siRNA-treated DCs (group C), $100 \mu 1$ 1.0x107/ml MUC1-CRT-primed 4T1-treated DCs (group D) or $100 \mu \mathrm{l} 1.0 \times 10^{7} / \mathrm{ml} \mathrm{SOCS1} \mathrm{siRNA-treated}$ and MUC1-CRT-primed 4T1-treated (double-treated) DCs (group E). One day post-injection, $50 \mu \mathrm{g}$ TNF- $\alpha$ was intraperitoneally administered to each mouse. Tumor size $\left(\mathrm{mm}^{3}\right)$ was assessed every other day. After 16 days, mice were sacrificed, tumors were isolated and the size of the tumors was measured.

To assess the production of cytokines from BALB/c mice bearing $4 \mathrm{~T} 1$ tumors, tumors were collected and depleted of red blood cells, and IFN- $\gamma$ and TNF- $\alpha$ production was detected as aforementioned.

Statistical analysis. Comparisons between two groups were assessed with a Mann-Whitney U test. Comparisons among multiple groups were performed using one-way analysis of variance followed by Tukey's post hoc analysis. Kaplan-Meier survival curves were analyzed using log-rank tests. GraphPad Prism software (version 5.0; GraphPad Software, Inc., La Jolla, CA, USA) was used to create graphs and to perform statistical analysis. $\mathrm{P}<0.05$ was considered to indicate a statistically significant difference.

\section{Results}

SOCS1 negatively regulates DCs in vitro. To investigate whether SOCS1 negatively regulates DCs in vitro, three synthetic siRNA oligo duplexes were efficiently transfected into the DC2.4 cell line using Lipofectamine 2000. As verified by western blot analysis, when compared with $\mathrm{NC}$ group the protein expression levels of SOCS1 in DC2.4 cells transfected with SOCS1 siRNA2 were significantly reduced $(\mathrm{P}<0.001$ vs. NC; Fig. 1A and B), which were significantly lower than those in the cells transfected with SOCS1 siRNA1 and siRNA3, respectively ( $\mathrm{P}<0.05$ vs. SOCS1 siRNA2; Fig. 1A and B). In addition, when compared with $\mathrm{NC}$ group the cells transfected with SOCS1 siRNA2 pSTAT1 expression levels were significantly increased ( $\mathrm{P}<0.001$ vs. NC; Fig. $1 \mathrm{~A}$ and $\mathrm{C})$, and the secretion of cytokine IFN- $\gamma$ following LPS stimulation was significantly increased ( $\mathrm{P}<0.01$ vs. NC; Fig. 1D). These data suggested that SOCS1 siRNA2 negatively regulated the JAK/STAT pathway and activated pro-inflammatory cytokine production. Therefore, SOCS1 siRNA2 was used for subsequent experiments.

Cellular uptake of hpp10-DRBD-SOCS1 siRNA. To validate the penetration of BMDCs and the delivery of SOCS1 siRNA by the recombinant protein hpp10-DRBD (Fig. 2A), a cellular uptake experiment was performed. Fluorescence microscopy was used to demonstrate transfection efficiency and revealed that $>95 \%$ of the DCs were transfected successfully with SOCS1 siRNA (data not shown). Cells were subsequently collected and lysed for western blot analysis. The results demonstrated that hpp10-DRBD successfully delivered siRNA into BMDCs, and that this silenced the expression of SOCS1 (Fig. 2B).

Silencing of SOCS1 enhances the immunological effect of MUC1-CRT-primed 4T1-treated DCs in vitro. To validate whether SOCS1 siRNA enhances cytokine production in MUC1-CRT-primed 4T1-treated BMDCs, BMDCs were incubated with SOCS1 siRNA followed by MUC1-CRT-primed 4T1 cells prior to being incubated with splenocytes. BMDCs were transfected successfully with MUC1-CRT (data not shown). Compared with NC group, the double-treated BMDCs exhibited significantly increased production of IFN- $\gamma$ and TNF- $\alpha(\mathrm{P}<0.001$ vs. NC; Fig. $3 \mathrm{~A}$ and $\mathrm{B})$, and production of these molecules was also significantly increased compared with MUC1-CRT-primed 4T1-treated BMDCs ( $\mathrm{P}<0.05$ vs. SOCS1 siRNA2; Fig. 3A and B). These results demonstrated that SOCS1-silencing, combined with MUC1-CRT-primed 4T1 cell treatment, may induce increased cytokine production by DCs.

The induction of $\mathrm{T}$ cell proliferation by BMDCs is an important antitumor function. As presented in Fig. 3C, compared with $\mathrm{NC}$ group the double-treatment of BMDCs significantly induced $\mathrm{T}$ cell production $(\mathrm{P}<0.001 \mathrm{vs}$. NC) and this induction was significantly increased compared with the MUC1-CRT-primed 4T1-treated BMDCs ( $\mathrm{P}<0.05$; Fig. 3C), suggesting that SOCS1-silencing, followed by MUC1-CRT-primed 4T1 treatment of DCs, increased T cell proliferation.

Subsequently, the cytotoxicity of pre-treated BMDCs on the breast cancer 4T1 cell line was evaluated via an LDH release assay. As presented in Fig. 3D, compared with NC group the double-treatment of BMDCs significantly induced LDH release ( $\mathrm{P}<0.001$ vs. $\mathrm{NC}$ ), and the cytotoxic effect of the double treatment was increased compared with MUC1-CRT-primed 4T1-treated BMDCs ( $<<0.05$ vs. SOCS1 siRNA2; Fig. 3D). These results suggested that SOCS1-silencing, followed by MUC1-CRT-primed 4T1 treatment of DCs, is associated with a higher level of membrane disturbance, indicating cytotoxicity. Collectively, the experiments indicated that silencing of SOCS1 in MUC1-CRT-primed 4T1-treated BMDCs induced immunological effects in vitro.

Silencing of SOCS1 enhances the immunological effects of MUC1-CRT-primed 4T1-treated DCs in vivo. The in vitro experimental data demonstrated that SOCS1-silencing combined with MUC1-CRT-primed 4T1 treatment of BMDCs may serve as a novel therapy for targeting cancer cells. To assess this in vivo, a breast cancer-bearing BALB/c mouse model was generated and subjected to pre-treated BMDCs that were developed in different ways, as before mentioned (groups A-E). Growth curves of tumors were produced from daily caliper measurements (Fig. 4A and B). The mice were sacrificed on the final day and the volume and weight of the excised tumors were measured (Fig. 4C-E). Compared with 
NC group the double-treated BMDCs significantly inhibited the growth of $4 \mathrm{~T} 1$ cells $(\mathrm{P}<0.01$ vs. NC; Fig. 4D and $\mathrm{E})$ and the double-treated BMDCs had a greater inhibitory effect compared withtheMUC1-CRT-primed 4T1-treated BMDCs $(\mathrm{P}<0.05$ vs. SOCS1 siRNA2; Fig. 4D and E).

Furthermore, tumors were collected and depleted of red blood cells, and IFN- $\gamma$ and TNF- $\alpha$ production was detected. As presented in Fig. 4F and G, compared with NC group the double-treated BMDCs exhibited significantly increased production of IFN- $\gamma$ and TNF- $\alpha(\mathrm{P}<0.001$ vs. NC; Fig. 4F and $\mathrm{G})$, and this was also significantly increased compared with that in the MUC1-CRT-primed 4T1-treated BMDCs $(\mathrm{P}<0.05$ vs. SOCS1 siRNA2; Fig. 4F and G). These experiments indicated that the silencing of SOCS1 combined with MUC1-CRT-primed 4T1 treatment of BMDCs may induce enhanced immunological effects in vivo.

\section{Discussion}

DCs are considered to be the most effective APCs with regard to their ability to prime tumor antigen-specific $\mathrm{T}$ cells and initiate immune responses against tumors (6). DC-based tumor vaccines represent a promising therapeutic approach, but have achieved limited success in clinical settings thus far. In order to improve the efficiency of DC maturation, our previous studies used CRT to translocate MUC1 to the 4T1 cell surface, where they worked together to induce a strong apoptotic reaction. The results demonstrated that the exposure of MUC1-CRT on the surface of breast cancer 4T1 cells facilitates their uptake by DCs and the subsequent presentation of TAAs to T cells (10).

DC maturation serves as the critical switch from the maintenance of self-tolerance to the induction of immunity. However, the identification of a means of overcoming inhibitory immune regulatory mechanisms and eliciting effective T-cell responses to antigens preferentially expressed by tumor cells remains a major challenge. SOCS1 acts as a critical 'brake' in DCs that disables their potency as a tumor vaccine. SOCS1 functions as a negative regulator of signaling by various cytokines; for example, Hanada et al (17) observed that $\mathrm{SOCS}^{-/}$DCs exhibited a more mature phenotype, were hyper-responsive to LPS and induced auto-reactive antibody production. Furthermore, Shen et al (11) demonstrated that SOCS1 serves a critical function in regulating the extent of DC antigen presentation and hence the magnitude of adaptive immunity. Additionally, a previous study revealed that silencing of SOCS1 in antigen-presenting DCs enhances antigen-specific antitumor immunity (11).

The present study attempted to further increase the potency of MUC1-CRT-primed 4T1-treated DCs by using siRNA to silence the expression of an endogenous molecule in DCs. To begin with, a SOCS1 siRNA with high knockdown efficiency in the DC2.4 cell line was selected (Fig. 1) and, subsequently, hpp10-DRBD was utilized to deliver this siRNA into BMDCs. The use of other materials for delivery, including cationic lipids and polymers, often fails to successfully introduce siRNAs into the entire population of cells, particularly primary cells and non-adherent cells, and such materials may also produce cytotoxic effects when used at high concentrations. By contrast, the hpp10 delivery peptide has been revealed to rapidly transport various types of biomolecules into the entire population of cells in our previous study (14). In addition, DRBD is able to mask the negative charge of siRNA, allowing hpp10 to efficiently deliver the siRNA into the DCs (15). In the present study, hpp10-DRBD was demonstrated to be highly efficient in facilitating the internalization of SOCS1 siRNA by BMDCs (Fig. 2).

The results of the present study demonstrated that SOCS1 regulated the extent of antigen presentation by mature DCs, thereby providing a regulatory mechanism that allows DCs to control the magnitude and duration of adaptive immunity (Fig. 3). Vaccination with SOCS1-silenced and MUC1-CRT-primed 4T1-treated BMDCs enhances antigen-specific antitumor immunity (Fig. 4), and SOCS1 silencing may permit antigen-presenting immunogenic DCs to persistently stimulate antigen-specific T cells in vivo (14).

\section{Acknowledgements}

The authors would like to thank the Nature Science Foundation of China (grant nos. 81201766 and 81550028), the Hubei Office of Education Foundation (grant no. Q20151204), the Hubei Office of Education Foundation (grant nos. Q20151204 and B2016022) and the Yi Chang Office of Education Foundation (grant no. A16-301-14) for providing financial support.

\section{References}

1. Obeid M, Tesniere A, Ghiringhelli F, Fimia GM, Apetoh L, Perfettini JL, Castedo M, Mignot G, Panaretakis T, Casares N, et al: Calreticulin exposure dictates the immunogenicity of cancer cell death. Nat Med 13: 54-61, 2007.

2. Obeid M, Tesniere A, Panaretakis T, Tufi R, Joza N, van Endert P, Ghiringhelli F, Apetoh L, Chaput N, Flament C, et al: Ecto-calreticulin in immunogenic chemotherapy. Immunol Rev 220: 22-34, 2007.

3. Ahn YH, Hong SO, Kim JH, Noh KH, Song KH, Lee YH, Jeon JH, Kim DW, Seo JH and Kim TW: The siRNA cocktail targeting interleukin 10 receptor and transforming growth factor- $\beta$ receptor on dendritic cells potentiates tumour antigen-specific CD8(+) T cell immunity. Clin Exp Immunol 181: 164-178, 2015.

4. Steinman RM, Hawiger D and Nussenzweig MC: Tolerogenic dendritic cells. Annu Rev Immunol 21: 685-711, 2003.

5. Chen L: Co-inhibitory molecules of the B7-CD28 family in the control of T-cell immunity. Nat Rev Immunol 4: 336-347, 2004.

6. Hodi FS, Mihm MC, SoifferRJ,Haluska FG, Butler M, Seiden MV, Davis T, Henry-Spires R, MacRae S, Willman A, et al: Biologic activity of cytotoxic T lymphocyte-associated antigen 4 antibody blockade in previously vaccinated metastatic melanoma and ovarian carcinoma patients. Pro Natl Acad Sci USA 100: 4712-4717, 2003

7. Wang J, Gao ZP, Qin S, Liu CB and Zou LL: Calreticulin is an effective immunologic adjuvant to tumor associate antigen. Exp Ther Med 14: 3399-3406, 2017.

8. Demaria O, Gassart A, Coso S, Gestermann N, Di Domizio J, Flatz L, Gaide O, Michielin O, Hwu P, Petrova TV, et al: STING activation of tumor endothelial cells initiates spontaneous and therapeutic antitumor immunity. Proc Natl Acad Sci USA 112: 15408-15413, 2015.

9. Ghosh S, Sarkar M, Ghosh T, Guha I, Bhuniya A, Saha A, Dasgupta S, Batik S, Bose A and Baral R: Neem leaf glycoprotein promotes dual generation of central and effector memory CD8(+) T cells against sarcoma antigen vaccine to induce protective anti-tumor immunity. Mol Immunol 71: 42-53, 2016.

10. Gardai SJ, McPhilips KA, Frasch SC, Janssen WJ, Starefeldt A, Murphy-Ullrich JE, Bratton DL, Oldenborg PA, Michalak M and Henson PM: Cell-surface calreticulin initiates clearance of viable or apoptotic cells through trans-activation of LRP on the phagocyte. Cell 123: 321-334, 2005. 
11. Shen L, Evel-Kabler K, Strube R and Chen SY: Silencing of SOCS1 enhances antigen presentation by dendritic cells and antigen-specific anti-tumor immunity. Nat Biotechnol 22: 1546-1553, 2004.

12. Kim JH, Kang TH, Noh KH, Kim SH, Lee YH, Kim KW, Bae HC, Ahn YH, Choi EY, Kim JS, et al: Enhancement of DC vaccine potency by activating the PI3K/AKT pathway with a small interfering RNA targeting PTEN. Immunol Lett 134: 47-54, 2010.

13. Kim JH, Kang TH, Noh KH, Bae HC, Ahn YH, Lee YH, Choi EY, Chun KH, Lee SJ and Kim TW: Blocking the immunosuppressive axis with small interfering RNA targeting interleukin (IL)-10 receptor enhances dendritic cell-based vaccine potency. Clin Exp Immunol 165: 180-189, 2011.

14. Wang H, Ma JL, Yang YG, Song Y, Wu J, Qin YY, Zhao XL, Wang J, Zou LL, Wu JF, et al: Efficient therapeutic delivery by a novel cell-permeant peptide derived from KDM44 protein for antitumor and antifibrosis. Oncotarget 7: 49075-49090, 2016.

15. Eguchi A, Meade BR, Chang YC, Fredrickson CT, Willert K, Puri N and Dowdy SF: Efficient siRNA delivery into primary cells by a peptide transduction domain-ds RNA binding domain fusion protein. Nat Biotechnol 27: 567-571, 2009.
16. Yang H, Li S, Li F, Yu K, Yang F and Xiang J: Recombinant expression of a modified shrimp anti-lipopolysaccharide factor gene in Pichia pastoris GS115 and its characteristic analysis. Mar Drugs 14: E152, 2016.

17. Hanada T, Yoshida H, Kato S, Tanaka K, Masutani K, Tsukada J, Nomura Y, Mimata H, Kubo M and Yoshimura A: Suppressor of cytokine signaling-1 is essential for suppressing dendritic cell activation and system autoimmunity. Immunity 19: 437-450, 2003.

18. Kumar A, Kundu S and Debnath DM: Expression, purification and evaluation of recombinant lipoprotein of Salmonella typhi as a vaccine candidate. Biologicals 46: 108-113, 2017.

c) () $(-)$ This work is licensed under a Creative Commons International (CC BY-NC-ND 4.0) License. 